Article

\title{
On Bilateral Contractions
}

\author{
Chi-Ming Chen ${ }^{1, *}$, Gholamreza Heidary Joonaghany ${ }^{2}$, Erdal Karapınar ${ }^{3, *}$ and \\ Farshid Khojasteh 4 (D)
}

1 Institute for Computational and Modeling Science, National Tsing Hua University, 521 Nan-Dah Road, Hsinchu City 30013 , Taiwan

2 Department of Mathematics, Arak Branch, Islamic Azad University, Arak 38361-1-9131, Iran; ghheidari@iau-arak.ac.ir

3 Department of Medical Research, China Medical University Hospital, China Medical University, Taichung 40402, Taiwan

4 Young Researcher and Elite Club, Arak Branch, Islamic Azad University, Arak 38361-1-9131, Iran; fr_khojasteh@yahoo.com

* Correspondence: chenchiming@mx.nthu.edu.tw (C.-M.C.); karapinar@mail.cmuh.org.tw or erdalkarapinar@yahoo.com (E.K.)

Received: 11 May 2019; Accepted: 10 June 2019; Published: 12 June 2019

\begin{abstract}
In this manuscript, we introduce a new type of contraction, bilateral contraction which merges two significant approaches in the fixed point theory: Caristi type contractions and Jaggi type contractions. The principal aim of the main result is to enrich the literature by combining the techniques of the mentioned two celebrated results that belong to Jaggi and Caristi. We consider an example to indicate the validity and genuine nature of the main result.
\end{abstract}

Keywords: Ćirić type contraction; Caristi fixed point theorem; fixed point; contraction

MSC: 46T99; 47H10; 54H25

\section{Introduction and Preliminaries}

Fixed point theory is based on the existence and uniqueness of the solution of a simple equation $T x=x$ where $T$ is a self-mapping on a non-empty set. This equation can be restated as $F(x)=0$ where $F(x)=x-T x$. Despite the simple problem statement, finding a solution of this equation is very difficult and sometimes impossible. The first affirmative response to this problem was given by Banach [1] under certain conditions: when $T$ forms contractive mapping and $X$ is equipped with a norm so that the corresponding topology yields completeness. In 1930, Caccioppoli [2] observed the analog of Banach's theorem in the setting of complete metric spaces. There are several extensions and generalizations of the Banach-Caccioppoli theorem with similar proofs. On the other hand, in 1976 Caristi [3] published a fixed point theorem whose proof and statement are quite different from the Banach-Caccioppoli theorem and its consequence.

Most of the metric fixed theorems consist of a linear combination of the distances between two distinct points $x, y$ in a metric space $(X, d)$ and their images. More precisely, there are six distances, $d(x, y), d(x, T y), d(T x, y), d(x, T x),(T y, y)$ and $d(T x, T y)$, and linear correlations of them; such as, Banach-Caccioppoli $d(T x, T y) \leq k d(x, y)$; Kannan $d(T x, T y) \leq \frac{k}{2}[d(x, T x)+d(T y, y)]$; Chatterjea $d(T x, T y) \leq \frac{k}{2}[d(y, T x)+d(T y, x)] ;$ Ćirić-Reich-Rus $d(T x, T y) \leq \frac{k}{3}[d(x, y)+d(x, T x)+d(T y, y)] ;$ and, so on, where $k \in[0,1)$. One of the initial nonlinear forms were given by Jaggi [4] and Dass-Gupta [5] who used rational forms in their fixed point equations and hence fixed point theorems.

In this note, we aim to merge and revisit the renowned fixed point results of both Caristi [3] and Jaggi [4]. For this purpose, we introduce the notion of a Jaggi type bilateral contraction. In addition, 
we strive to unify the celebrated fixed point theorems of Dass-Gupta [5] and Caristi [3] by introducing the notion of a Dass-Gupta type bilateral contraction. After proving that these contractions possess fixed points, we express illustrative examples. We note that this work can be thought as a continuation of [6].

\section{Main Result}

We start this section by giving the definition of a Jaggi type bilateral contraction that is inspired from the renowned results of Jaggi [4].

Definition 1. A self-mapping $T$ on metric space $(X, d)$ is called a Jaggi type bilateral contraction if there is a $\vartheta: X \rightarrow[0, \infty)$ such that

$$
d(x, T x)>0 \text { implies } d(T x, T y) \leq(\vartheta(x)-\vartheta(T x)) R_{T}(x, y),
$$

for all distinct $x, y \in X$, where

$$
R_{T}(x, y)=\max \left\{d(x, y), \frac{d(x, T x) \cdot d(y, T y)}{d(x, y)}\right\}
$$

Theorem 1. Suppose that $T$ is continuous and forms a Jaggi type bilateral contraction on a complete metric $(X, d)$. Then, $T$ possesses at least a fixed point.

Proof. We start the proof by taking an arbitrator point $x \in X$ and rename it as $x_{0}:=x$. Note that if $T x_{0}=x_{0}$, the proof is completed. We shall construct an iterative sequence $\left\{x_{n}\right\}$ by using the Picard definition $x_{n+1}=T x_{n}$ for each non-negative integer $n$. Without loss of generality, we presume that

$$
d\left(x_{n}, x_{n+1}\right)=d\left(x_{n}, T x_{n}\right)>0 \text { for all non-negative integer } n .
$$

It is clear that in the converse case, if there exists a positive integer $m$ such that $x_{m}=x_{m+1}=T x_{m}$, then the proof of is over.

From the expression (2), we guarantee that the inequality (1) is applicable for the sequence $\left\{x_{n}\right\}$. For that simplicity, we let $\delta_{n}=d\left(x_{n}, x_{n-1}\right)$ and employ (1), then we get

$$
\begin{aligned}
\delta_{n+1} & =d\left(x_{n}, x_{n+1}\right)=d\left(T x_{n-1}, T x_{n}\right) \\
& \leq\left(\vartheta\left(x_{n-1}\right)-\vartheta\left(T x_{n-1}\right)\right) R_{T}\left(x_{n-1}, x_{n}\right) \\
& =\left(\vartheta\left(x_{n-1}\right)-\vartheta\left(x_{n}\right)\right) \max \left\{d\left(x_{n}, x_{n-1}\right), d\left(x_{n}, x_{n+1}\right)\right\} \\
& =\left(\vartheta\left(x_{n-1}\right)-\vartheta\left(x_{n}\right)\right) \max \left\{\delta_{n}, \delta_{n+1}\right\} .
\end{aligned}
$$

Now, we consider the cases $\max \left\{\delta_{n}, \delta_{n+1}\right\}=\delta_{n}$ and $\max \left\{\delta_{n}, \delta_{n+1}\right\}=\delta_{n+1}$ separately.

Case 1: Suppose $\max \left\{\delta_{n}, \delta_{n+1}\right\}=\delta_{n}$. On account of (3) and also (2), we find

$$
\delta_{n+1}=d\left(x_{n}, x_{n+1}\right)=d\left(T x_{n-1}, T x_{n}\right) \leq\left(\vartheta\left(x_{n-1}\right)-\vartheta\left(x_{n}\right)\right) d\left(x_{n}, x_{n-1}\right)=\left(\vartheta\left(x_{n-1}\right)-\vartheta\left(x_{n}\right)\right) \delta_{n} .
$$

Accordingly, we get,

$$
0<\frac{\delta_{n+1}}{\delta_{n}} \leq \vartheta\left(x_{n-1}\right)-\vartheta\left(x_{n}\right) \text { for each } n \in \mathbb{N}
$$


So we conclude that the sequence $\left\{\vartheta\left(x_{n}\right)\right\}$ is not only non-increasing but also necessarily positive. Consequently, the mentioned sequence converges to some $\ell \geq 0$. On the other hand, for each $n \in \mathbb{N}$, we find

$$
\begin{aligned}
\sum_{k=1}^{n} \frac{\delta_{k+1}}{\delta_{k}} & \leq \sum_{k=1}^{n}\left[\vartheta\left(x_{k-1}\right)-\vartheta\left(x_{k}\right)\right] \\
& =\left(\vartheta\left(x_{0}\right)-\vartheta\left(x_{1}\right)\right)+\left(\vartheta\left(x_{1}\right)-\vartheta\left(x_{2}\right)\right)+\ldots+\left(\vartheta\left(x_{n-1}\right)-\vartheta\left(x_{n}\right)\right) \\
& =\vartheta\left(x_{0}\right)-\vartheta\left(x_{n}\right) \rightarrow \vartheta\left(x_{0}\right)-\ell<\infty, \text { as } n \rightarrow \infty .
\end{aligned}
$$

In other words, we obtain

$$
\sum_{n=1}^{\infty} \frac{\delta_{n+1}}{\delta_{n}}<\infty
$$

Accordingly, we have

$$
\lim _{n \rightarrow \infty} \frac{\delta_{n+1}}{\delta_{n}}=0 .
$$

On account of (4), for $\kappa \in(0,1)$, there exists $n_{0} \in \mathbb{N}$ such that

$$
\frac{\delta_{n+1}}{\delta_{n}} \leq \kappa,
$$

for all $n \geq n_{0}$. It yields that

$$
d\left(x_{n+1}, x_{n}\right) \leq \kappa d\left(x_{n}, x_{n-1}\right),
$$

for all $n \geq n_{0}$.

Case 2: Now, suppose that $\max \left\{\delta_{n}, \delta_{n+1}\right\}=\delta_{n+1}$. Regarding (3) we find

$$
d\left(x_{n+1}, x_{n}\right) \leq\left(\vartheta\left(x_{n-1}\right)-\vartheta\left(x_{n}\right)\right) d\left(x_{n+1}, x_{n}\right),
$$

and since $\left\{\vartheta\left(x_{n}\right)\right\}$ is non-increasing and positive sequence, and so converges to some $\ell \geq 0$ (see the similar argument in Case 1) thus we have $1 \leq\left(\vartheta\left(x_{n-1}\right)-\vartheta\left(x_{n}\right)\right) \rightarrow 0$, as $n \rightarrow \infty$ and this is a contradiction.

This contradiction also concludes the same result in Case 1 that the sequence $\left\{\delta_{n}\right\}$ is non-increasing sequence.

In what follows we claim that the sequence $\left\{x_{n}\right\}$ converges to some $\omega \in X$.

Combining the observation in Case 1, Case 2, and (5) we deduce that the sequence $\left\{d\left(x_{n+1}, x_{n}\right)\right\}$ is non-increasing and bounded below. Consequently, it is convergent to some $\delta^{*} \geq 0$. Since $\kappa<1$, it is easily verified that $\delta^{*}=0$.

On the other hand, for each $m, n \in \mathbb{N}$ with $m>n$, we have

$$
d\left(x_{n}, x_{m}\right) \leq \sum_{k=n}^{m-1} d\left(x_{i}, x_{i+1}\right) \leq \frac{\kappa^{n}}{1-\kappa} d\left(x_{0}, x_{1}\right) .
$$

Thus, we have $\lim \sup _{n \rightarrow \infty}\left\{d\left(x_{n}, x_{m}\right): m>n\right\}=0$. As a result, $\left\{x_{n}\right\}$ is a Cauchy sequence. On account of the fact that $X$ is complete, there exists $\omega \in X$ such that $x_{n}$ converges to $\omega$.

As a next step, we assert that $\omega$ is the fixed point of $T$. Since $T$ is continuous, we find $T \omega=T\left(\lim _{n \rightarrow \infty} x_{n}\right)=\lim _{n \rightarrow \infty} T x_{n}=\lim _{n \rightarrow \infty} x_{n+1}=\omega$.

Inspired the results of Dass-Gupta [5], we introduce the notion of a Dass-Gupta type bilateral contraction as follows: 
Definition 2. A self-mapping $T$ on metric space $(X, d)$ is called a Dass-Gupta type bilateral contraction if there is $a \vartheta: X \rightarrow[0, \infty)$ such that

$$
d(x, T x)>0 \text { implies } d(T x, T y) \leq(\vartheta(x)-\vartheta(T x)) Q_{T}(x, y)
$$

for all $x, y \in X$, where

$$
Q_{T}(x, y)=\max \left\{d(x, y), \frac{(1+d(x, T x)) \cdot d(y, T y)}{1+d(x, y)}\right\}
$$

Theorem 2. Suppose that $T$ forms a Dass-Gupta type bilateral contraction on a complete metric $(X, d)$. Then, $T$ possesses at least a fixed point.

Proof. We mainly follow the steps of the proof of Theorem 1. By verbatim, we construct a sequence $\left\{x_{n}\right\}$ so that

$$
d\left(x_{n}, x_{n+1}\right)=d\left(x_{n}, T x_{n}\right)>0 \text { for all non-negative integer } n .
$$

Indeed, in case of existing a positive integer $m$ such that $x_{m}=x_{m+1}=T x_{m}$ then, the proof of is over.

On account of the expression (8), we can utilize the inequality (7) with the sequence $\left\{x_{n}\right\}$. Set $\delta_{n}=d\left(x_{n}, x_{n-1}\right)$ and employ (7), then we get

$$
\begin{aligned}
\delta_{n+1} & =d\left(x_{n}, x_{n+1}\right)=d\left(T x_{n-1}, T x_{n}\right) \\
& \leq\left(\vartheta\left(x_{n-1}\right)-\vartheta\left(T x_{n-1}\right)\right) Q_{T}\left(x_{n-1}, x_{n}\right) \\
& =\left(\vartheta\left(x_{n-1}\right)-\vartheta\left(x_{n}\right)\right) \max \left\{d\left(x_{n-1}, x_{n}\right), \frac{\left(1+d\left(x_{n-1}, T x_{n-1}\right)\right) \cdot d\left(x_{n}, T x_{n}\right)}{1+d\left(x_{n-1}, x_{n}\right)}\right\} \\
& =\left(\vartheta\left(x_{n-1}\right)-\vartheta\left(x_{n}\right)\right) \max \left\{\delta_{n}, \delta_{n+1}\right\} .
\end{aligned}
$$

Regarding the similarity with the structures of the proof of Theorem 1, after then, by following the corresponding lines in the proof of Theorem 1 , we deduce that $\left\{x_{n}\right\}$ is a Cauchy sequence. On account of the fact that $X$ is complete, there exists $\omega \in X$ such that $x_{n}$ converges to $\omega$.

Unlike the statement of Theorem 1 , the continuity of the given mapping $T$ is not assumed. As a next step, we assert and prove that $\omega$ is the fixed point of $T$ without continuity assumption. Assume, on the contrary, that $w$ is not a fixed point of $T$, that is $d(\omega, T \omega)>0$. On account of the observation $d(\omega, T \omega)>0$, we employ the inequality (7) that yields

$$
\begin{aligned}
d(\omega, T \omega) & \leq d\left(\omega, x_{n+1}\right)+d\left(x_{n+1}, T \omega\right) \\
& =d\left(\omega, x_{n+1}\right)+d\left(T x_{n}, T \omega\right) \\
& =d\left(\omega, x_{n+1}\right)+\left(\vartheta\left(x_{n}\right)-\vartheta\left(T x_{n}\right)\right) Q_{T}\left(x_{n}, \omega\right) \\
& =d\left(\omega, x_{n+1}\right)+\left(\vartheta\left(x_{n}\right)-\vartheta\left(x_{n+1}\right)\right) \max \left\{d\left(x_{n}, \omega\right), \frac{\left(1+d\left(x_{n}, T x_{n}\right)\right) d(\omega, T \omega)}{1+d\left(x_{n}, \omega\right)}\right\} \\
& =d\left(\omega, x_{n+1}\right)+\left(\vartheta\left(x_{n}\right)-\vartheta\left(x_{n+1}\right)\right) \max \left\{d\left(x_{n}, \omega\right), \frac{\left(1+d\left(x_{n}, x_{n+1}\right)\right) d(\omega, T \omega)}{1+d\left(x_{n}, \omega\right)}\right\}
\end{aligned}
$$

Since the sequences $\left\{\vartheta\left(x_{n}\right)\right\}$ tends to $r \geq 0$, for sufficiently large $n \in \mathbb{N}$, we have

$$
d(\omega, T \omega) \leq \lim _{n \rightarrow \infty}\left(d\left(\omega, x_{n+1}\right)+\left(\vartheta\left(x_{n}\right)-\vartheta\left(x_{n+1}\right)\right)\right) d(\omega, T \omega)=0 .
$$

Consequently, we obtain $d(\omega, T \omega)=0$, that is, $T \omega=\omega$.

From Theorem 1, we get the corresponding result for complete metric spaces. The following example shows that the Theorem 1 is not a consequence of Banach's contraction principle. 
Example 1. Let $X=\{0,1,2\}$ endowed with the following metric:

$$
d(0,1)=1, d(2,0)=1, d(1,2)=\frac{3}{2} \text { and } d(a, a)=0, \forall a \in X d(a, b)=d(b, a) \forall a, b \in X
$$

Let $T(0)=0, T(1)=2, T(2)=0$. Define $\vartheta: X \rightarrow[0, \infty)$ as $\vartheta(2)=2, \vartheta(0)=0, \vartheta(1)=4$. Thus for all $x \in X$ such that $d(x, T x)>0$, (in this example, $x \neq 0$ ), we have

$$
\begin{aligned}
& d(T 1, T 2) \leq(\vartheta(1)-\vartheta(T(1))) R_{T}(2,1), \\
& d(T 2, T 1) \leq(\vartheta(2)-\vartheta(T(2))) R_{T}(2,1), \\
& d(T 1, T 0) \leq(\vartheta(1)-\vartheta(T(1))) R_{T}(1,0), \\
& d(T 2, T 0) \leq(\vartheta(2)-\vartheta(T(2))) R_{T}(2,0) .
\end{aligned}
$$

Thus the mapping $T$ satisfies our condition and also has a fixed point. Note that $d(T 1, T 0)=d(1,0)$. Thus, it does not satisfy Banach contraction principle.

Example 2. Let $X=\{O=(1,1), A=(1,2), B=(3,3)\}$ be endowed with the metric d defined by

$$
d\left(\left(x_{1}, x_{2}\right),\left(y_{1}, y_{2}\right)\right)=\left|x_{1}-y_{1}\right|+\left|x_{2}-y_{2}\right| \text {. }
$$

It is easy to see that $(X, d)$ is a complete metric space.

Suppose that $T: X \rightarrow X$ is defined as follows:

$$
T(1,1)=(3,3), T(1,2)=(1,1) \text { and } T(3,3)=(3,3) .
$$

Also, define $\vartheta: X \rightarrow[0, \infty)$ as follows:

$$
\vartheta(1,1)=2, \vartheta(1,2)=4 \text { and } \vartheta(3,3)=0 \text {. }
$$

We have:

$$
\begin{array}{llll}
d(T(1,1), T(1,2))=4, & \vartheta(1,1)-\vartheta(T(1,1))=2, & R_{T}((1,1),(1,2))=4, & Q_{T}((1,1),(1,2))=\frac{5}{2} \\
d(T(1,1), T(3,3))=0, & \vartheta(1,1)-\vartheta(T(1,1))=2, & R_{T}((1,1),(3,3))=4, & Q_{T}((1,1),(3,3))=4 \\
d(T(1,2), T(1,1))=4, & \vartheta(1,2)-\vartheta(T(1,2))=2, & R_{T}((1,2),(1,1))=4, & Q_{T}((1,2),(1,1))=4 \\
d(T(1,2), T(3,3))=4, & \vartheta(1,2)-\vartheta(T(1,2))=2, & R_{T}((1,2),(3,3))=3 & Q_{T}((1,2),(3,3))=3 .
\end{array}
$$

So, for all distinct $x, y \in X$,

$$
d(x, T x)>0 \text { implies } d(T x, T y) \leq(\vartheta(x)-\vartheta(T x)) R_{T}(x, y),
$$

where

$$
R_{T}(x, y)=\max \left\{d(x, y), \frac{d(x, T x) \cdot d(y, T y)}{d(x, y)}\right\} .
$$

Additionally, for all $x, y \in X$,

$$
d(x, T x)>0 \text { implies } d(T x, T y) \leq(\vartheta(x)-\vartheta(T x)) R_{T}(x, y),
$$


where

$$
Q_{T}(x, y)=\max \left\{d(x, y), \frac{(1+d(x, T x)) \cdot d(y, T y)}{1+d(x, y)}\right\} .
$$

Thus the mapping $T$ satisfies in all the conditions of Theorems 1 and 2 as well. Note that $d(T(1,2), T(3,3))>d((1,2),(3,3))$, so it does not satisfy the Banach contraction principle.

In what follows, we state some of immediate consequence as the main results.

Hereinafter, we presume that $T$ is a self-mapping on a complete metric $(X, d)$ in all the corollaries below, without mentioning them again and again.

Corollary 1. If there is a $\vartheta: X \rightarrow[0, \infty)$ such that

$$
d(x, T x)>0 \text { implies } d(T x, T y) \leq(\vartheta(x)-\vartheta(T x))\left[a_{1} d(x, y)+a_{2} \frac{d(x, T x) d(y, T y)}{d(x, y)}\right]
$$

for all distinct $x, y \in X$, where $a_{1}, a_{2}$ are non-negative real numbers with a sum 1 and $T$ is continuous. Then, $T$ has at least a fixed point.

Corollary 2. If there is $a \vartheta: X \rightarrow[0, \infty)$ such that

$$
d(x, T x)>0 \text { implies } d(T x, T y) \leq(\vartheta(x)-\vartheta(T x)) \frac{d(x, T x) d(y, T y)}{d(x, y)}
$$

for all distinct $x, y \in X$, where $T$ is continuous. Then, $T$ has at least a fixed point.

Hereinafter, we presume that $T$ is a self-mapping on a complete metric space $(X, d)$ in all the corollaries below, without mentioning them again and again.

Corollary 3. If there is $a \vartheta: X \rightarrow[0, \infty)$ such that

$$
d(x, T x)>0 \text { implies } d(T x, T y) \leq(\vartheta(x)-\vartheta(T x))\left[a_{1} d(x, y)+a_{2} \frac{(1+d(x, T x)) d(y, T y)}{1+d(x, y)}\right]
$$

for all $x, y \in X$, where $a_{1}, a_{2}$ are non-negative real numbers with a sum 1 . Then, $T$ has at least a fixed point.

Corollary 4. If there is a $\vartheta: X \rightarrow[0, \infty)$ such that

$$
d(x, T x)>0 \text { implies } d(T x, T y) \leq(\vartheta(x)-\vartheta(T x)) d(x, y)
$$

for all $x, y \in X$. Then, $T$ has at least a fixed point.

Corollary 5. If there is $a \vartheta: X \rightarrow[0, \infty)$ such that

$$
d(x, T x)>0 \text { implies } d(T x, T y) \leq(\vartheta(x)-\vartheta(T x)) \frac{(1+d(x, T x)) d(y, T y)}{1+d(x, y)}
$$

for all $x, y \in X$. Then, $T$ has at least a fixed point.

Sketch of the proofs of the stated corollaries above. The direct proof of the corollaries are derived by verbatim of Theorem 1 . On the other hand, by using the following inequalities we derive the desired results in corollaries above.

1. $\left[a_{1} d(x, y)+a_{2} \frac{d(x, T x) d(y, T y)}{d(x, y)}\right] \leq R_{T}(x, y)$,

2. $\frac{d(x, T x) d(y, T y)}{d(x, y)} \leq R_{T}(x, y)$, 
3. $d(x, y) \leq Q_{T}(x, y)$,

4. $\quad\left[a_{1} d(x, y)+a_{2} \frac{(1+d(x, T x)) d(y, T y)}{1+d(x, y)}\right] \leq Q_{T}(x, y)$,

5. $\quad \frac{(1+d(x, T x)) d(y, T y)}{1+d(x, y)} \leq Q_{T}(x, y)$,

Author Contributions: All authors contributed equally and significantly in writing this article. All authors read and approved the final manuscript.

Funding: This research received no external funding.

Conflicts of Interest: The authors declare no conflict of interest.

\section{References}

1. Banach, S. Sur les opérations dans les ensembles abstraits et leur application aux équations intégrales. Fundam. Math. 1922, 3, 133-181. [CrossRef]

2. Caccioppoli, R. Una teorema generale sull'esistenza di elementi uniti in una transformazione funzionale. Ren. Accad. Naz Lincei 1930, 11, 794-799.

3. Caristi, J. Fixed point theorems for mappings satisfying inwardness conditions. Trans. Am. Math. Soc. 1976, 215, 241-251. [CrossRef]

4. Jaggi, D.S. Some unique fixed point theorems. Indian J. Pure Appl. Math. 1977, 8, 223-230.

5. Dass, B.K.; Gupta, S. An extension of Banach contraction principle through rational expression. Indian J. Pure Appl. Math. 1975, 6, 1455-1458.

6. Karapinar, E.; Khojasteh, F.; Mitrovic, Z.D. A Proposal for Revisiting Banach and Caristi Type Theorems in b-Metric Spaces. Mathematics 2019, 7, 308. [CrossRef]

(C) 2019 by the authors. Licensee MDPI, Basel, Switzerland. This article is an open access article distributed under the terms and conditions of the Creative Commons Attribution (CC BY) license (http://creativecommons.org/licenses/by/4.0/). 